【研究简报】

\title{
高灵敏度毛细管电泳-激光诱导荧光- 增强型电荷耦合检测器
}

\author{
马明生 吴晓军 刘利琳 刘国诠* \\ (中国科学院化学研究所, 北京 100080 .*联系人)
}

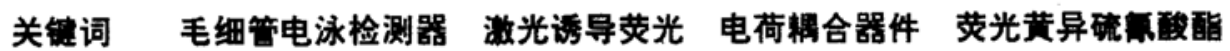

激光诱导荧光检测器 (LIF) 是目前灵敏度最高的毛细管电泳 (CE) 检测法 ${ }^{[1]}$ 之一, 常用的 测光器件主要有光电倍增管、二极管阵列检测器 ${ }^{[2]}$ 和电荷耦合器件 (CCD). CCD 具有较低的 读出噪声、较小的暗电流、较高的量子效率和较宽的动态范围, 有可能用于发展高性能的 LIF 检测系统. Timperman 等 ${ }^{[3]}$ 采用 $\mathrm{Ar} / \mathrm{Kr}$ 混合气体离子激光光源与 $\mathrm{CCD}$ 检测器相配合的方 法, 检测到 80 个硫代罗丹明 101 分子及 220 个荧光黄分子. 本工作中利用 binning 方式提高 增强型电荷耦合器件 (ICCD) 检测器灵敏度, 在优化条件下可检测 27 个荧光黄异硫呀酸酯(简 称 FITC)分子. 同时利用记录不同电泳峰的波谱可进行 CE-LIF 的定性分析.

\section{1 实验}

未经涂敷的熔融石英毛细管 $(65 \mathrm{~cm} \times 50 \mu \mathrm{mi}$ i.d., 河北永年光导纤维厂), 按毛细管区带 电泳 (CZE) 模式分离苂光黄异硫氧酸酯(简称 FITC, Fluorescein Isothiocyanate, Isomer I, Sig$\mathrm{ma}$, USA). 以 $20 \mathrm{mmol} / \mathrm{L}$ 四硼酸钠为缓冲液, 电泳工作电压 $20 \mathrm{kV}$ (RHR 30P60 Spellman, Plainview, NY); 重力进样法进样 $10 \mathrm{~s}$, 检测窗口位于距正极端 $35 \mathrm{~cm}$ 处. 通过透镜将 $488 \mathrm{~nm}$ 氯离子激光 (Spinnaker 1161-All $200 \mathrm{~mW}$ 输出功率可调, Spectra-Physics, USA)聚焦到毛细管 上, 为减少 Ralaiy 散射对荧光检测的影响, 入射激光束与毛细管呈 $30^{\circ}$ 角 ${ }^{1)}$. 用一 X10 消色散 物镜(Olympus, Japan) 在与毛细管和激光束所成平面垂直方向上接收苂光信号, 并通过一 X20 长焦物镜 (航天部 207 所, 北京) 将其聚焦到谱仪光棚上(Spectra Pro-275, Acton Research Co., USA, 光棚刻度 $150 / \mathrm{mm}$ ), 谱仪入射狭琏宽度为 $50 \mu \mathrm{m}$, 光栅中心波长 $660 \mathrm{~nm}$. 利用光 纤耦合增强型电荷耦合器件 $($ ICCD $)($ EEV576 $\times 384$, Princeton Instruments, Inc., USA) 在 510 527 nm 范围内监测荧光信号, 通过 CSMA 软件 (Princeton Instruments, Inc., USA)同时 监测和记录荧光光谱和毛细管电泳图谱, 并将其存入计算机中 (486MX 计算机, Dell Inc., USA). 于绘图仪上输出 (HP ColorPro 绘图仪, Hewlett Packard, USA). 另外, 置一 $488 \mathrm{~nm}$ 截止滤波片 (中国科学院电子研究所铰膜室)于两个物镜之间, 以消除散射光的影响.

\section{2 结果与讨论}

\section{1 通过 binning 方式提高检测器信啉比 $(S / N)$}

ICCD 的 binning 操作方式是先将若干个相邻象元中的电荷加和到一起, 形成一个大的象

1) 马明生, 吴晓军, 刘国诠. 增强型电荷耦合器件用于毛细管电泳激光诱导苂光的检测. 高等学校化学学报, 1997 (待发表). 
元, 然后再进行读出、放大和数值转换, 从 ，而进一步提高信噪比. 从理论上讲, 对微 弱信号进行检测, 通过 binning 方式将 $N$ 行象元加和到一起, 其 $S / N$ 较 binning 前 要提高 $N^{1 / 2}$ 倍 ${ }^{[4]}$. 图 1 中比较了采用 binning 方式将 6 行象元加和后和只读取其中 一行象元中的电荷对 $4.5 \times 10^{-14} \mathrm{~mol} / \mathrm{L}$ 的 FITC 检测结果, 其 $S / N$ 提高了 2.17 倍, 接近理论值 2.45. 这其中的差异可能是由 所加和的行象元间荧光强度的不一致性所 致.

在实际操作中所 binning 象元的行数 还受到 ICCD 井容 (well capacity) 的限制.

一般来讲, 移位寄存器的井容是 ICCD 片基 上的 2 3 倍, 因此, 当所 binning 的象元中

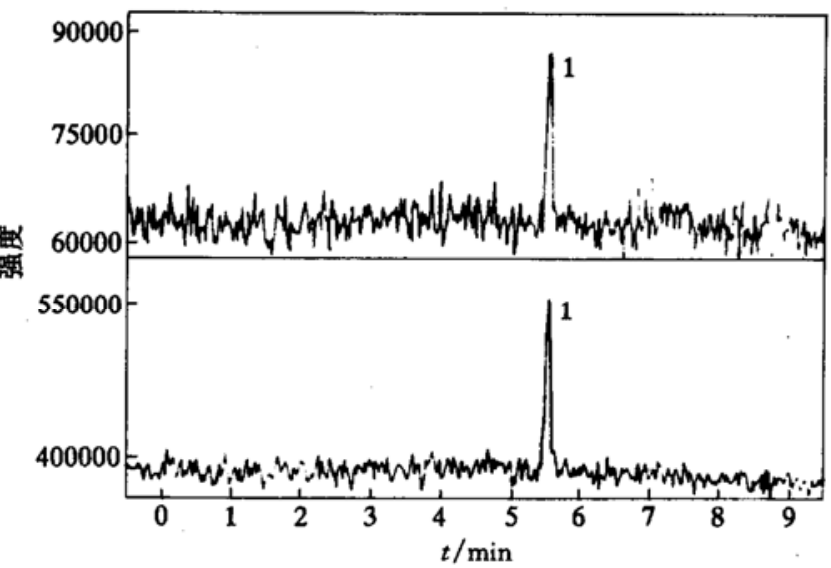

图 1 binning 前后 $S / N$ 比较

上图: 单行象元, 下图: binning 后 6 行象元加和. 分离电压: 20 $\mathrm{kV}$, 缓冲液: $20 \mathrm{mmol} / \mathrm{L}$ 四磞酸钢; 峰 1:FITC, 浓度 $4.5 \times 10^{-14}$ $\mathrm{mol} / \mathrm{L}$; 进样方式:重力进样 $10 \mathrm{~s}$, 进样高度: $8 \mathrm{~cm}$ 电荷数过大时, 将会超出 ICCD 的动态范围, 导致数据的丢失.

\section{2 检测灵敏度}

图 2 是利用重力进样 $10 \mathrm{~s}$ 钟时, 毛细管电泳分离 $4.5 \times 10^{-14} \mathrm{~mol} / \mathrm{L}$ 浓度 FITC 的 LIF-ICCD 电泳图. 图中 FITC 检测信噪比为 6 , 按 $S / N=3$ 估算, 本检测器的浓度检测限为 $2.25 \times$ $10^{-14} \mathrm{~mol} / \mathrm{L}$. 进样时的液面高度差为 $8 \mathrm{~cm}$, 根据 Poiseuille 公式 ${ }^{[5]}$ 计算进样体积约为 $2 \mathrm{~nL}$, 得 出质量检测限为 $4.5 \times 10^{-23} \mathrm{~mol}$, 即可检测到 27 个分子.

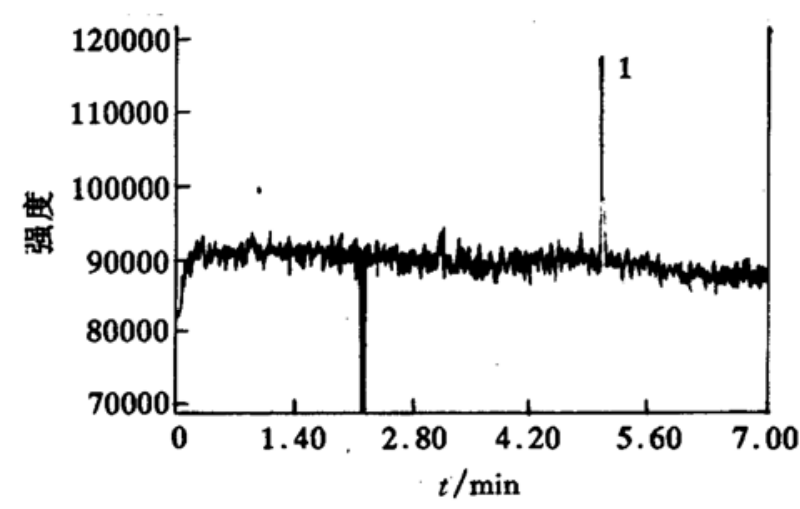

图 2 FITC 的毛细管电泳激光诱导荧光谱图 峰 1: FITC, 浓度: $4.5 \times 10^{-14} \mathrm{~mol} / \mathrm{L}$; 缓冲液: 20 $\mathrm{mmol} / \mathrm{L}$ 四硼酸钠, 分离电压: $20 \mathrm{kV}$; 进样方式:重力进 样 $10 \mathrm{~s}$, 进样高度: $8 \mathrm{~cm}$

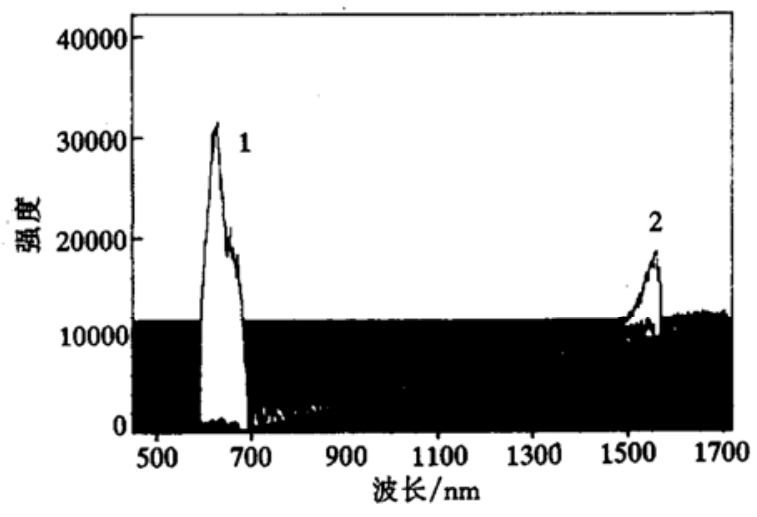

图 3 罗丹明 B 和 FITC 的时间-强度-波谱三维 毛细管电泳图

峰 1 : 罗丹明 B; 峰 2:FITC; 毛细管电泳条件与图 2 相同

\section{3 具有波谱分辩功能的监测模式}

在得到毛细管电泳图谱的同时还可以得到每个电泳峰的荧光光谱是用 ICCD 进行 CELIF 检测的另一特点. 图 3 显示了罗丹明 B 和 FITC 的时间-强度-波谱三维毛细管电泳图, 其 中罗丹明 $\mathrm{B}$ 的最大荧光强度出现在 $574.6 \mathrm{~nm}$ 处, FITC 的则在 $518.4 \mathrm{~nm}$ 处. 从图上可分辨 


\title{
科 学 通 报
}

\section{样条小波多重过滤的滤波分析}

\author{
邹小勇 莫金垣 \\ (中山大学化学系, 广州 510275)
}

\section{关链词阶跃伏安法 样条小波分析 多国过滤滤波}

在电分析化学研究过程中, 当被测物浓度很低, 采样数据序列常常含有非常严重的白噪 声, 严重影响检出限和输出波形, 同时也给伏安波的进一步分析带来困难. 在众多的提高信 噪比方法中, 以 Fourier 为基础的数字处理方法得到广泛的研究和应用, 它主要把时间域的测 量变换成频率域的测量, 但是, Fourier 方法有几个无法克服的弱点 ${ }^{[1]}$. 近几年来, 小波分析在 工程界和理论界获得了广泛的研究和应用. 它的主要特点为: 可以把信号按频率直接分解, 容 易求出信号频域分布状态的时域表示, 这种表示方法同时具有时频局部化的特点; 另外, 在某 一段频域范围(小波波谱)的信号,在不了解原始信号的情况下, 可选择适合于该曲线特性的小 波基,这样能更准确地处理所描述的信号.

本文提出的样条小波多重滤波分析,其滤波方式同样条小波单次滤波方式明显不同之处 在于:在滤波过程中,为了真实地提取各种相关信息,所采用的方法是把过滤后的 “滤液”噪声 当成原始信号进行重滤波处理, 并将之对有用信号进行补偿, 同时将本滤波方法同阶跃伏安法

出前面的峰为罗丹明 B, 后面的峰为 FITC.

\section{3 结论}

本文对 ICCD 用 binning 操作方式提高 CE-LIF 检测灵敏度方面进行了探讨．以 ICCD 为 检测器的 CE-LIF 系统对 FITC 的质量检测限达到 27 个分子, 同时也展示了 ICCD 检测器波 谱分辨功能在毛细管电泳荧光检测方面的应用前景.

致谢 本工作为国家科委和中国科学院计划局及国家自然科学基金(批准号: 29375215)资助 项目.

\section{参考文献}

1 Yeung E S, Wang P, Li W, et al. Laser fluorescence detector for capillary electrophoresis. J Chormatogr, 1992, 608: 73 77

2 Carson S, Cohen A S, Belenkii A, et al. DNA sequencing by capillary electrophoresis: use of a two-laser-two-window intensified diode array detection system. Anal Chem, 1993, 65: $3219 \sim 3226$

3. Timperman A T, Khatib K, Sweedler J V. Wavelength-resolved fluorescence detection in capillary electrophoresis. Anal Chem, 1995, 67: 139 144

4 Epperson P M, Denton M B. Binning spectral images in a charge-coupled device. Anal Chem, 1989, 61: $1513 \sim 1519$

5 Rose Jr D J, Jorgenson J W. Characterization and automation of sample introduction methods for capillary zone electrophoresis. Anal Chem, 1988, 60: 642 648 